\title{
A New ${ }^{18}$ F-Labeled Myocardial PET Tracer: Myocardial Uptake After Permanent and Transient Coronary Occlusion in Rats
}

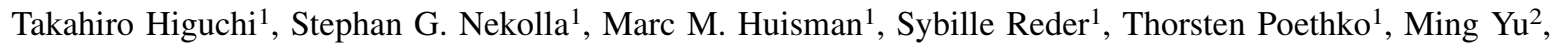 \\ Hans-Jurgen Wester ${ }^{1}$, David S. Casebier ${ }^{2}$, Simon P. Robinson ${ }^{2}$, Rene M. Botnar ${ }^{1}$, and Markus Schwaiger ${ }^{1}$ \\ ${ }^{1}$ Department of Nuclear Medicine, Klinikum Rechts der Isar, Technische Universität München, Munich, Germany; and ${ }^{2}$ Departments of \\ Discovery Chemistry and Discovery Biology, Bristol-Myers Squibb Medical Imaging, North Billerica, Massachusetts
}

\begin{abstract}
Conventional myocardial perfusion PET tracers require onsite tracer production because of their short radioactive half-lives. To investigate the potential of a new ${ }^{18} \mathrm{~F}$-labeled pyridazinone analog ( $\left.{ }^{18} \mathrm{~F}-\mathrm{BMS}-747158-02\right)$, we characterized this tracer in a rat model of permanent and transient coronary occlusion using small-animal PET. Methods: Myocardial ${ }^{18} \mathrm{~F}-\mathrm{BMS}-747158-02$ distribution in healthy rats $(n=7)$, rats with transient (3-min) left coronary artery occlusion $(n=11)$, and rats with permanent left coronary occlusion $(n=11)$ was analyzed with a dedicated small-animal PET scanner. Results: Normal hearts demonstrated intense and almost homogeneous tracer uptake throughout the left ventricle for more than $2 \mathrm{~h}$. During permanent coronary occlusion, PET demonstrated perfusion defects, which remained unchanged $(37.6 \% \pm 8.8 \%, 37.4 \% \pm 10.2 \%$, and $36.2 \% \pm 9.8 \%$ left ventricle at 15,45 , and $115 \mathrm{~min}$, respectively, after tracer injection). After transient ischemia, the induced defect size decreased significantly after reperfusion $(16.2 \% \pm$ $9.3 \%, 6.0 \% \pm 6.5 \%$, and $1.4 \% \pm 1.3 \%$ left ventricle). Tracer reinjection after transient ischemia resulted in normalization of the induced defect. Conclusion: Coronary occlusion yielded distinct myocardial ${ }^{18} \mathrm{~F}-\mathrm{BMS}-747158-02$ uptake defects in the area of ischemia, which demonstrated normalization of activity after reperfusion and reinjection. These promising kinetic parameters may allow for assessment of flow using exercise-rest protocols similar to those used in combination with exercise and rest perfusion SPECT.
\end{abstract}

Key Words: cardiology (clinical); PET; animal imaging; ${ }^{18} \mathrm{~F}$; infarction; ischemia; perfusion

J Nucl Med 2008; 49:1715-1722

DOI: 10.2967/jnumed.108.053967

$\mathbf{M}$ yocardial exercise-rest perfusion imaging using SPECT with ${ }^{201} \mathrm{Tl},{ }^{99 \mathrm{~m}} \mathrm{Tc}$-sestamibi, or ${ }^{99 \mathrm{~m}} \mathrm{Tc}$-tetrofosmine is widely applied for clinically diagnosing and determining

Received Apr. 29, 2008; revision accepted Jun. 24, 2008.

For correspondence or reprints contact: Takahiro Higuchi, Nuklearmedizinische Klinik der Technischen Universität München, Ismaninger Strasse 22, 81675 Munich, Germany.

E-mail: higuchi@po2.nsknet.or.jp

COPYRIGHT @ 2008 by the Society of Nuclear Medicine, Inc. the prognosis of ischemic heart disease (1). However, falsepositive findings caused by attenuation artifacts and underestimation of absolute perfusion abnormalities in patients with balanced 3-vessel disease are well-known limitations of the method (2). Moreover, the recent development of angiogenic therapies may require high-sensitivity, high-resolution imaging for monitoring changes in perfusion more subtle than those that occur with conventional therapies (3).

PET can overcome some limitations of SPECT by providing better temporal and spatial resolution, greater sensitivity, and established algorithms for attenuation correction $(4,5)$. There are 3 well-established tracers for myocardial PET perfusion imaging: ${ }^{82} \mathrm{Rb},{ }^{13} \mathrm{~N}$-ammonia, and ${ }^{15} \mathrm{O}$-water. These tracers have short radioactive half-lives of $76 \mathrm{~s}, 10 \mathrm{~min}$, and $2.1 \mathrm{~min}$, respectively. ${ }^{82} \mathrm{Rb}$ is produced by a radionuclide generator system, whereas production of ${ }^{13} \mathrm{~N}$-ammonia and ${ }^{15} \mathrm{O}$-water require an expensive on-site cyclotron. In addition, the short radioactive half-life requires imaging protocols with tracer injections while the patient is on the scanner. These temporal limitations make it difficult to perform the exercise stress examinations commonly used in SPECT myocardial perfusion imaging.

${ }^{18} \mathrm{~F}-$-labeled perfusion PET tracers, including ${ }^{18} \mathrm{~F}-\mathrm{BMS}-$ 747158-02, for the evaluation of myocardial perfusion have recently been introduced $(6-10) .{ }^{18} \mathrm{~F}$-labeled tracers with radioactive half-lives of $110 \mathrm{~min}$ overcome the half-life limitations of previously used PET perfusion tracers. Such tracers may be distributed by a central cyclotron facility in a manner similar to ${ }^{18} \mathrm{~F}$-FDG. Moreover, ${ }^{18} \mathrm{~F}$ provides better image quality and spatial resolution because it emits a lower-energy positron that travels a shorter distance in tissue before annihilation $(11,12)$. Initial reports of ${ }^{18} \mathrm{~F}$ BMS-747158-02 showed the compound to have promising properties as a new myocardial perfusion tracer, such as a specific, high myocardial extraction fraction and retention at different flow rates in rat and rabbit models $(8-10)$. However, whether the kinetics of ${ }^{18}$ F-BMS-747158-02 in normally perfused and transiently ischemic myocardium reflect suitability for clinical stress perfusion studies remains unknown. In this study, we aimed to further explore 
the potential clinical application of the novel PET tracer ${ }^{18}$ F-BMS-747158-02 for the diagnosis of coronary artery disease using permanent and transient coronary occlusion in rats and imaging with small-animal PET.

\section{MATERIALS AND METHODS}

${ }^{18}$ F-BMS-747158-02 was characterized using a small-animal PET system and rat models. ${ }^{18} \mathrm{~F}-\mathrm{BMS}-747158-02$ uptake in the myocardium of healthy rats was assessed. For comparison, ${ }^{13} \mathrm{~N}$-ammonia uptake in other healthy rats was studied. Furthermore, myocardial ${ }^{18}$ F-BMS-747158-02 uptake and time-dependent changes in tracer distribution were assessed in rats with permanent or transient coronary occlusion. One rat was studied with ${ }^{13} \mathrm{~N}$-ammonia PET and ${ }^{18} \mathrm{~F}-\mathrm{BMS}-747158-02$ PET $1 \mathrm{wk}$ after permanent coronary artery occlusion.

Experimental protocols were approved by the regional governmental commission of animal protection (Regierung von Oberbayern, Germany) and conformed with the guidelines of the U.S. National Institutes of Health (13).

\section{Tracer Production}

Tracer production was performed as described elsewhere (8-10).

\section{Animal Preparation}

Male Wistar rats weighing 250-300 g were used in all experiments. Healthy rats $(n=7)$, rats with permanent left coronary artery (LCA) occlusion $(n=11)$, and rats with transient LCA occlusion ( $n=11$ ) were studied (Fig. 1$)$. Before the intervention, the rats were anesthetized with intramuscularly administered midazolam $(0.1 \mathrm{mg} / \mathrm{kg})$, fentanyl $(1 \mu \mathrm{g} / \mathrm{kg})$, and medetomidine $(10 \mu \mathrm{g} / \mathrm{kg})$. The rats were mechanically ventilated and underwent thoracotomy.

In rats with permanent LCA occlusion, a 7-0 polypropylene suture on a small, curved needle was passed through the myocardium beneath the LCA and ligated to occlude the LCA (14). Coronary occlusion was confirmed by the regional cyanosis of the myocardial surface.

In the animals with transient LCA occlusion, the suture was passed through the myocardium beneath the LCA. Subsequently, both suture ends were passed through a small vinyl tube to make a snare. The suture material was pulled tightly against the vinyl tube to occlude the LCA. After a 3-min occlusion, reperfusion was obtained by release of the snare and confirmed by a myocardial blush over the area at risk (15). After the surgery, the chest was closed, and the rats were moved to the PET scanner.

\section{${ }^{18}$ F-BMS-747158-02 PET Protocols}

Five series of experiments (protocols A-E) were performed (Fig. 1).

Protocol A was designed to address the myocardial distribution of ${ }^{18} \mathrm{~F}-\mathrm{BMS}-747158-02$ in normal rats over $120 \mathrm{~min}$ after tracer injection $(n=5)$. Thirty-seven megabecquerels of ${ }^{18} \mathrm{~F}$-BMS747158-02 were injected into the tail vein just before the start of PET data acquisition.

Protocol B evaluated the effect of coronary occlusion. The rats were injected with ${ }^{18}$ F-BMS-747158-02 (37 MBq) 1 min after permanent occlusion of the LCA and imaged from 10 to $120 \mathrm{~min}$ after tracer injection $(n=5)$.

Protocol $\mathrm{C}$ was designed to analyze the tracer redistribution in a reperfusion model. The LCA was occluded for a period of $3 \mathrm{~min}$, and $37 \mathrm{MBq}$ of ${ }^{118} \mathrm{~F}-\mathrm{BMS}-747158-02$ were injected $1 \mathrm{~min}$ after

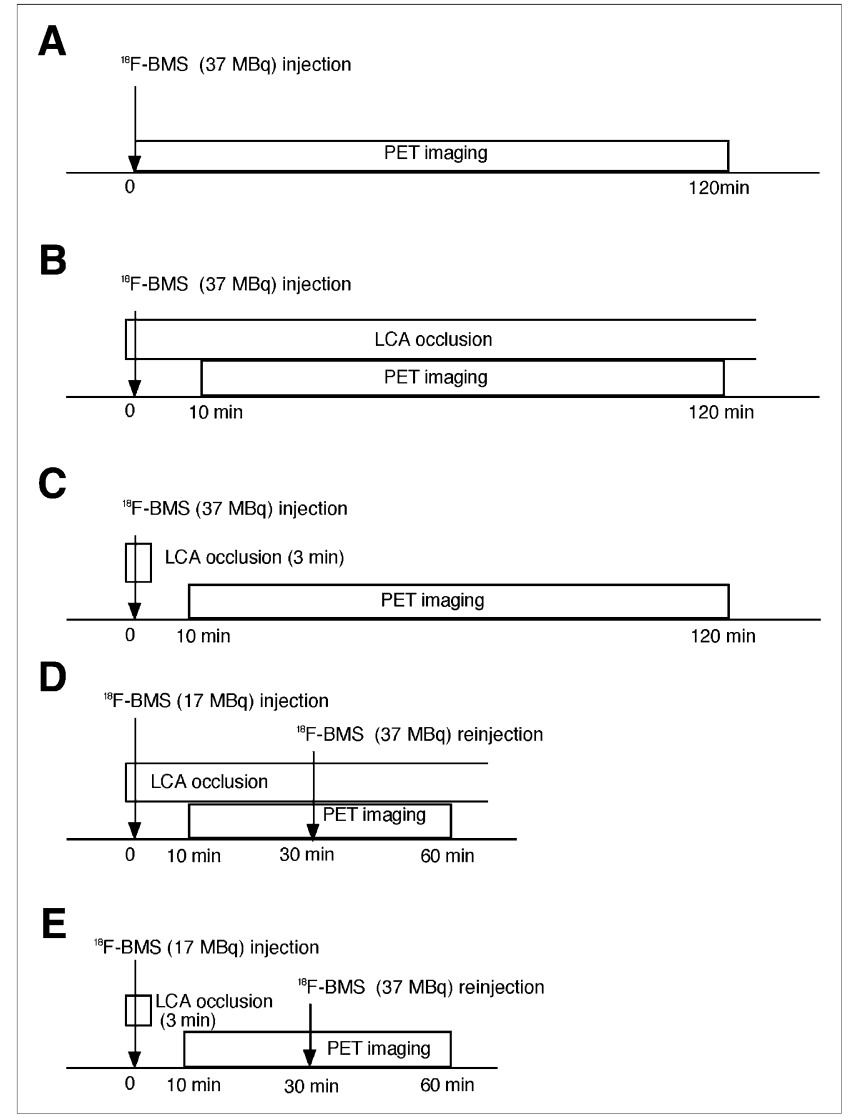

FIGURE 1. Schemas of experimental protocols of permanent and transient coronary occlusion and imaging: normal heart $(A)$, permanent LCA occlusion (B), transient LCA occlusion (C), reinjection after permanent LCA occlusion (D), and reinjection after transient LCA occlusion (E). ${ }^{18} \mathrm{~F}-\mathrm{BMS}={ }^{18} \mathrm{~F}-\mathrm{BMS}-747158-02$.

occlusion of the LCA. Image acquisition continued from 10 to 120 min after tracer injection $(n=5)$.

Protocols D and E were designed to investigate the effects of tracer reinjection on the cardiac tracer distribution in permanent (D) $(n=6)$ or transient (E) $(n=6)$ coronary occlusion. In both protocols, ${ }^{18} \mathrm{~F}-\mathrm{BMS}-747158-02$ was injected as described in protocols $\mathrm{B}$ and $\mathrm{C}$ at $10 \mathrm{~min}$ before the start of image acquisition (17 $\mathrm{MBq}$ ) and $30 \mathrm{~min}$ after the start of image acquisition (37 MBq), which continued for $60 \mathrm{~min}$.

\section{${ }^{13} \mathrm{~N}-$ Ammonia PET of Healthy Rats and Coronary Occlusion Model}

${ }^{13} \mathrm{~N}$-ammonia PET was performed on 8 healthy rats. ${ }^{13} \mathrm{~N}$ ammonia (55 MBq) was injected, and data were acquired from 5 to 15 min after tracer injection.

One rat was imaged $1 \mathrm{wk}$ after permanent occlusion of the LCA with both ${ }^{13} \mathrm{~N}$-ammonia and ${ }^{18} \mathrm{~F}-\mathrm{BMS}-747158-02$ PET. Imaging was performed for $10 \mathrm{~min}$ after a 5-min delay after injection of 55 $\mathrm{MBq}$ of ${ }^{13} \mathrm{~N}$-ammonia. After a delay of $40 \mathrm{~min}$ to allow for radioactive decay, $37 \mathrm{MBq}$ of ${ }^{18} \mathrm{~F}-\mathrm{BMS}-747158-02$ were administered, and a 10-min PET acquisition was performed.

\section{PET Acquisition and Reconstruction}

All PET was performed with the animals prone. A dedicated small-animal PET scanner was used (microPET Focus 120; 
Siemens Medical Solutions). Data were acquired in list-mode format and reconstructed into a dynamic image with 10-min frames for ${ }^{18} \mathrm{~F}-\mathrm{BMS}-747158-02$ PET and a single frame for ${ }^{13} \mathrm{~N}-$ ammonia PET.

The reconstruction was done using filtered backprojection with a cutoff at the Nyquist frequency, and the resulting images had $128 \times 128 \times 95$ voxels with a voxel size of $0.43 \times 0.43 \times 0.80 \mathrm{~mm}$. Data were normalized and corrected for randoms, dead time, and decay.

\section{Data Analysis}

The PET images were analyzed with the ASIPro software package (Siemens Medical Solutions) and the Munich Heart software package $(14,16)$.

Regions of interests were manually defined in a mid coronal slice at the heart (anterior wall) and in the blood pool (left ventricle), lung (right), and liver using ASIPro software. Percentages of injected dose $/ \mathrm{cm}^{3}$ of tissue in the heart region of interest and the ratio of tracer activity between the heart and other organs were calculated.

Volumetric sampling was applied to delineate 3-dimensional tracer distributions throughout the left ventricular myocardium, and tracer concentrations at each sampling point were displayed in a polar map using Munich Heart software $(14,16)$. Mean percentage uptake (expressed as a percentage of maximal uptake) was calculated for 5 segments in normal rat hearts. Tracer-uptake defects were characterized as having less than $50 \%$ activity when compared with the point of maximum activity in the myocardium. The mean percentage uptakes and regional defects were evaluated at 15,45 , and 115 min after tracer injection.

\section{Statistical Analysis}

All results were expressed as mean \pm SD. Statistical analysis was done with StatMate III (ATMS Co., Ltd.). Uptake ratio was compared between 2 groups by means of the Mann-Whitney test. Differences between group comparisons were identified using ANOVA followed by the Scheffé multiple-contrast hypothesis test. A value of $P$ less than 0.05 was considered statistically significant.

\section{RESULTS}

\section{F-BMS-747158-02 Distribution in Normal Rat Hearts}

Figure 2 demonstrates in vivo analysis of ${ }^{18} \mathrm{~F}$-BMS747158-02 uptake in healthy rats. The left ventricular myo-
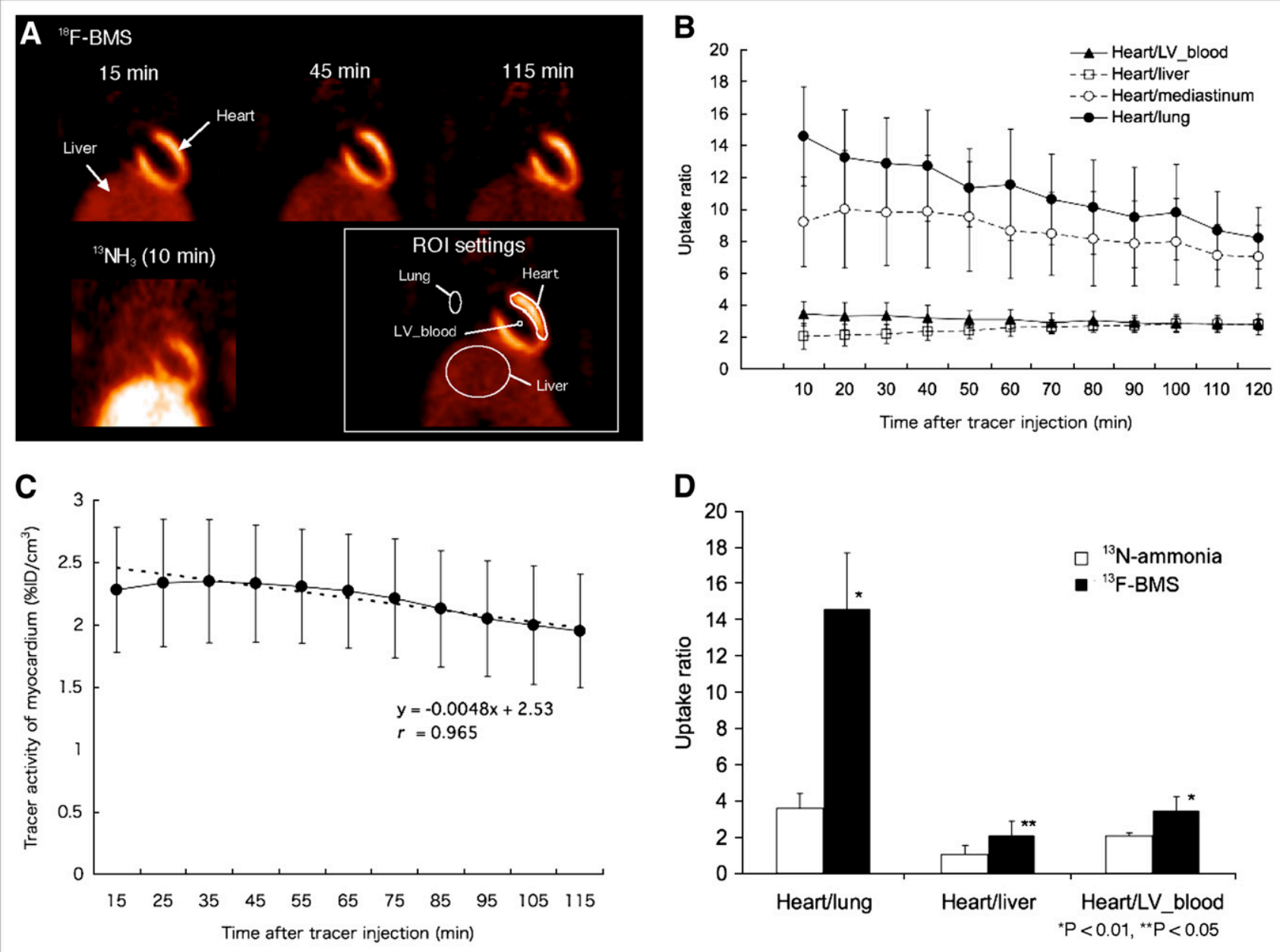

FIGURE 2. (A) Examples of ${ }^{18} \mathrm{~F}-\mathrm{BMS}-747158-02$ ( $\left.{ }^{18} \mathrm{~F}-\mathrm{BMS}\right)$ PET images of chest of healthy rat at 15 , 45 , and $115 \mathrm{~min}$ after tracer injection and ${ }^{13} \mathrm{~N}$-ammonia PET image at $10 \mathrm{~min}$ in coronal view. Example of regions of interest for measurement of tracer activity is displayed in white box. (B and $\mathrm{C}$ ) Ratio of ${ }^{18} \mathrm{~F}-\mathrm{BMS}$ uptake between myocardium and surrounding organs $(\mathrm{B})$ and absolute ${ }^{18} \mathrm{~F}-$ BMS activity of myocardium $\left(\% \mathrm{ID} / \mathrm{cm}^{3}\right)$ (C). (D) Comparison of ${ }^{18} \mathrm{~F}-\mathrm{BMS}$ uptake ratios at 15 min after injection and ${ }^{13} \mathrm{~N}$-ammonia at $10 \mathrm{~min}$. 


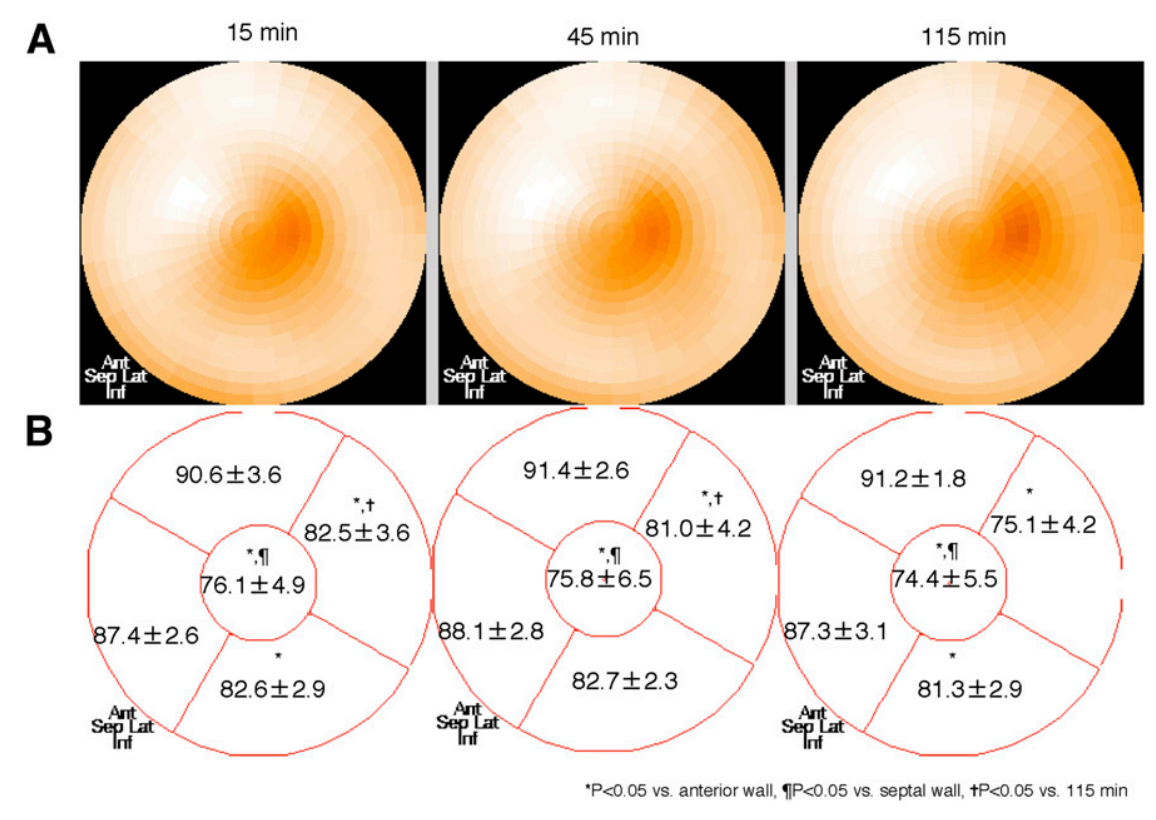

FIGURE 3. Normal pattern of left ventricular ${ }^{18} \mathrm{~F}-\mathrm{BMS}-747158-02$ ( $\left.{ }^{18} \mathrm{~F}-\mathrm{BMS}\right)$ distribution at 15,45 , and 115 min after tracer injection as shown in mean polar maps $(A)$ and as mean uptake values (\% left ventricle maximum) with SD (B). Uptake was nearly homogeneous throughout left ventricle, with slightly lower uptake in apical segment.

cardium showed excellent contrast to surrounding tissues in all rats. High tracer accumulation was preserved in the myocardium throughout the imaging period of $2 \mathrm{~h}$ (percentage injected dose [\% ID] $/ \mathrm{cm}^{3}>2.0$ from 15 to $115 \mathrm{~min}$ ), with a slight decrease from $30 \mathrm{~min}$ (Fig. 2C). The half-life of myocardial tracer activity on PET images was calculated as 265 min using linear fitting of the time-activity curve from 25 to $115 \min (y=-0.0048 x+2.53, r=0.965 ; y=$ tracer activity $\left[\% \mathrm{ID} / \mathrm{cm}^{3}\right], x=$ time after tracer injection $\left.[\mathrm{min}]\right)$. Time-dependent changes in tracer distribution in the heart and other organs were observed. Heart-to-lung ratio and heart-to-left ventricle blood-pool ratio decreased over time (heart-to-lung ratio from $14.6 \pm 3.10$ at $15 \mathrm{~min}$ to $8.70 \pm 2.4$ at $115 \mathrm{~min}$; heart-to-left ventricle blood pool ratio from $3.47 \pm 0.76$ at $15 \mathrm{~min}$ to $2.83 \pm 0.54$ at $115 \mathrm{~min}$ ) and heart-toliver ratio increased (from $2.07 \pm 0.80$ at $15 \mathrm{~min}$ to $2.82 \pm$ 0.39 at $115 \mathrm{~min}$ ) (Figs. 2A and 2B). These ratios, when compared with ${ }^{13} \mathrm{~N}$-ammonia (heart-to-lung, $3.60 \pm 0.80$; heart-to-liver, $1.05 \pm 0.49$; heart-to-left ventricle blood pool, $2.08 \pm 0.16$ ), show significantly improved myocardium-totissue contrast (Figs. 2A and 2D).

${ }^{18}$ F-BMS-747158-02 uptake in the myocardium was almost homogeneous throughout the left ventricle. However, small differences were observed, with the lowest uptake in apical segments, when compared with anterior, septal, inferior, and lateral areas at 15, 45, and $115 \mathrm{~min}$ after tracer injection. Small (approximately 5\%) but significant differences in tracer distribution were found in the lateral wall at $115 \mathrm{~min}$, as compared with 15 and $45 \mathrm{~min}$ (Fig. 3).

\section{F-BMS-747158-02 Uptake in LCA Occlusion}

${ }^{18}$ F-BMS-747158-02 PET images demonstrated a defect in the myocardium of all rats with LCA occlusion at $15 \mathrm{~min}$ after tracer injection. As shown in Figure 4, the induced ${ }^{18} \mathrm{~F}-\mathrm{BMS}-747158-02$ uptake defect visualized at $15 \mathrm{~min}$ corresponded precisely to the defect in ${ }^{13} \mathrm{~N}$-ammonia images. Images obtained using ${ }^{18} \mathrm{~F}-\mathrm{BMS}-747158-02$ demonstrated improved contrast and higher resolution resulting in better delineation of induced lesions, despite a higher injected dose of ${ }^{13} \mathrm{~N}$-ammonia $\left(57 \mathrm{MBq}\right.$ ) versus ${ }^{18} \mathrm{~F}-$ BMS-747158-02 (37 MBq).

\section{F-BMS-747158-02 Redistribution After Reperfusion}

The defect size induced by permanent LCA occlusion did not change over $2 \mathrm{~h}(37.6 \% \pm 8.8 \%$ left ventricle at 15 $\min , 37.4 \% \pm 10.2 \%$ left ventricle at $45 \mathrm{~min}$, and $36.2 \% \pm$ $9.8 \%$ left ventricle at $115 \mathrm{~min}$ ). The tracer activity (\% ID/ $\mathrm{cm}^{3}$ ) in normal myocardium and the induced defect were also constant throughout the imaging period. In contrast,

FIGURE 4. Short-axis images of rat heart 1 wk after coronary occlusion using ${ }^{18} \mathrm{~F}-\mathrm{BMS}-747158-02$ ( $\left.{ }^{18} \mathrm{~F}-\mathrm{BMS}\right)$ PET and ${ }^{13} \mathrm{~N}$-ammonia PET. Arrows indicate localization of myocardial infarction.

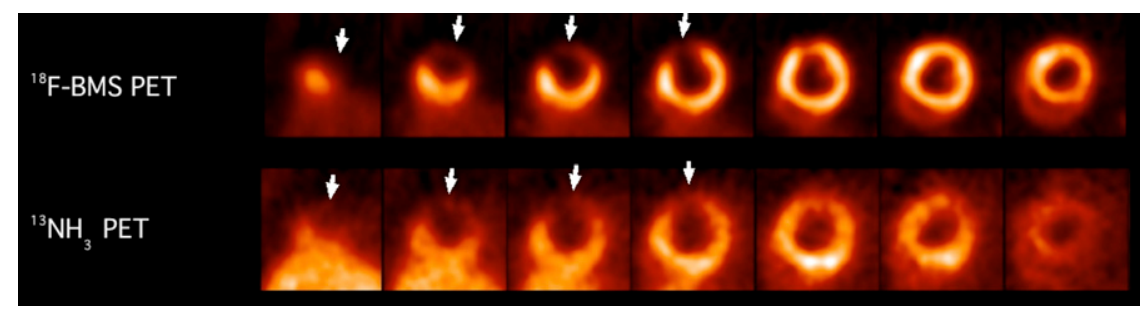


tracer redistributed in the induced defect after reperfusion after $3 \mathrm{~min}$ of coronary occlusion (Fig. 5). A significant reduction $(P<0.01)$ in perfusion defect size occurred after reperfusion $(16.2 \% \pm 9.3 \%$ left ventricle at $15 \mathrm{~min}$, $6.0 \% \pm 6.5 \%$ left ventricle at $45 \mathrm{~min}$, and $1.4 \% \pm 1.3 \%$ left ventricle at $115 \mathrm{~min}$ ). Tracer activity in the defect increased over time after reperfusion, whereas the activity of normally perfused myocardium remained constant. In rats with transient LCA occlusion (3 min) and reperfusion, the activity within the defect approached normal myocardial activity at 115 min after tracer injection (Fig. 6).

Tracer reinjection did not change the defect size $(29.7 \% \pm$ $10.8 \%$ at $15 \mathrm{~min}, 30.5 \% \pm 10.8 \%$ after reinjection) in animals with permanent LCA occlusion. In contrast, rats with transient LCA occlusion demonstrated resolution of the induced defect, with increased tracer activity after reinjection (defect size: $15.0 \% \pm 5.5 \%$ at $15 \mathrm{~min}, 0 \% \pm$ $0 \%$ after reinjection) (Fig. 7). The defect took $2 \mathrm{~h}$ to disappear without reinjection (protocol $\mathrm{C}$ ), but tracer reinjection enhanced the normalization process, which became apparent rapidly after reinjection in the transient LCA occlusion model (protocol E).

\section{DISCUSSION}

The results of this study indicate promising properties for ${ }^{18}$ F-BMS-747158-02 as a novel ${ }^{18} \mathrm{~F}$-labeled PET tracer for the diagnosis and evaluation of coronary artery disease. In the normal rat heart, tracer uptake in the left ventricular wall shows high contrast to surrounding tissue and high myocardial retention throughout the imaging period of $2 \mathrm{~h}$. This finding confirms the previously reported high first-pass extraction and high myocardial retention in isolated perfused rat hearts (10). The high contrast ratio between myocardial and hepatic tissue represents a favorable feature for the evaluation of the inferior wall of the left ventricle, as compared with ${ }^{13} \mathrm{~N}$-ammonia. In animals with coronary occlusion, the induced defect was clearly delineated and

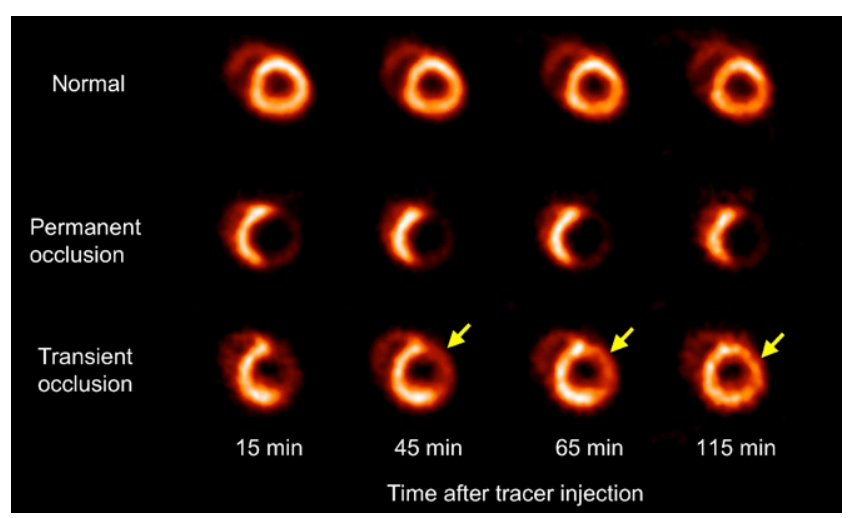

FIGURE 5. Representative short-axis images of rat hearts without coronary occlusion, permanent coronary occlusion, and transient coronary occlusion at different time points after tracer administration. Tracer redistribution is seen in induced defect after reperfusion (arrows). remained unchanged over the entire imaging protocol. In contrast, reperfusion after short, transient ischemia (3 min) yielded tracer redistribution to the induced defect. Tracer reinjection further enhanced the normalization process. The clinical application of this tracer for the diagnosis of coronary artery disease and for the assessment of tissue viability may involve tracer injection under physical stress conditions with early and late imaging studies.

PET has been proven to be a reliable and efficient technique to assess myocardial perfusion in patients with known or suspected coronary artery disease $(17,18)$. Recently, Bateman et al. compared the diagnostic accuracy of rest/pharmacologic stress perfusion with $99 \mathrm{~m}$ Tc-sestamibi SPECT and ${ }^{82} \mathrm{Rb}$ PET in matched patient populations (5). The results demonstrated higher overall accuracy for PET $(89 \%)$ than for SPECT (79\%), based on an angiographic criterion of more than $70 \%$ coronary stenosis. In patients with multivessel coronary disease, PET identified more defects than did SPECT (71\% vs. $48 \%)$.

Conventional PET perfusion tracers such as ${ }^{3} \mathrm{~N}$-ammonia, ${ }^{15} \mathrm{O}$-water, or ${ }^{82} \mathrm{Rb}$ have short physical half-lives, which allow repeated studies after decay. A pharmacologic stress protocol is commonly used for conventional PET perfusion studies because of technical limitations in performing exercise stress testing with short-lived isotopes. On the other hand, exercise stress testing is often preferred for SPECT perfusion imaging because it offers important clinical and hemodynamic information in addition to myocardial perfusion. Recently, Chow et al. compared treadmill exercise and dipyridamole stress using ${ }^{13} \mathrm{~N}$-ammonia perfusion PET (19). They found larger and more severe perfusion defects with physical exercise than with dipyridamole, indicating that exercise may offer diagnostic advantages for ${ }^{13} \mathrm{~N}$-ammonia perfusion imaging. Because of the longer half-life of ${ }^{18} \mathrm{~F}$, ${ }^{18}$ F-labeled perfusion tracers can potentially be used in exercise stress testing by injection of tracer during peak exercise and postinjection imaging. However, the longer half-life also limits the use of tracer in protocols involving repeated measurements on a single day. Therefore, protocols involving stress and rest studies on separate days, or validation of a tracer reinjection protocol, are required. In addition, an increased radiation dose should be considered when determining the injection dose of the tracer.

The chemical structure of BMS-747158-02, a modification of the pyridazinone insecticide pyidaben, was described in previous publications $(8-10)$. These compounds are known to bind tightly to mitochondrial complex I, with high affinity in myocardial tissue $(8,9,20)$. Observed highly selective myocardial tracer uptake may be explained partly by the high content of mitochondria in the left ventricular myocardium. Given the high extraction fraction, which appeared to be flow-independent as measured in isolated rat hearts (10), ${ }^{18} \mathrm{~F}-\mathrm{BMS}-747158-02$ uptake is most likely to reflect blood flow. The feasibility of quantification requires confirmation in large-animal models using microsphere reference measurements. In addition, the influence of hy- 


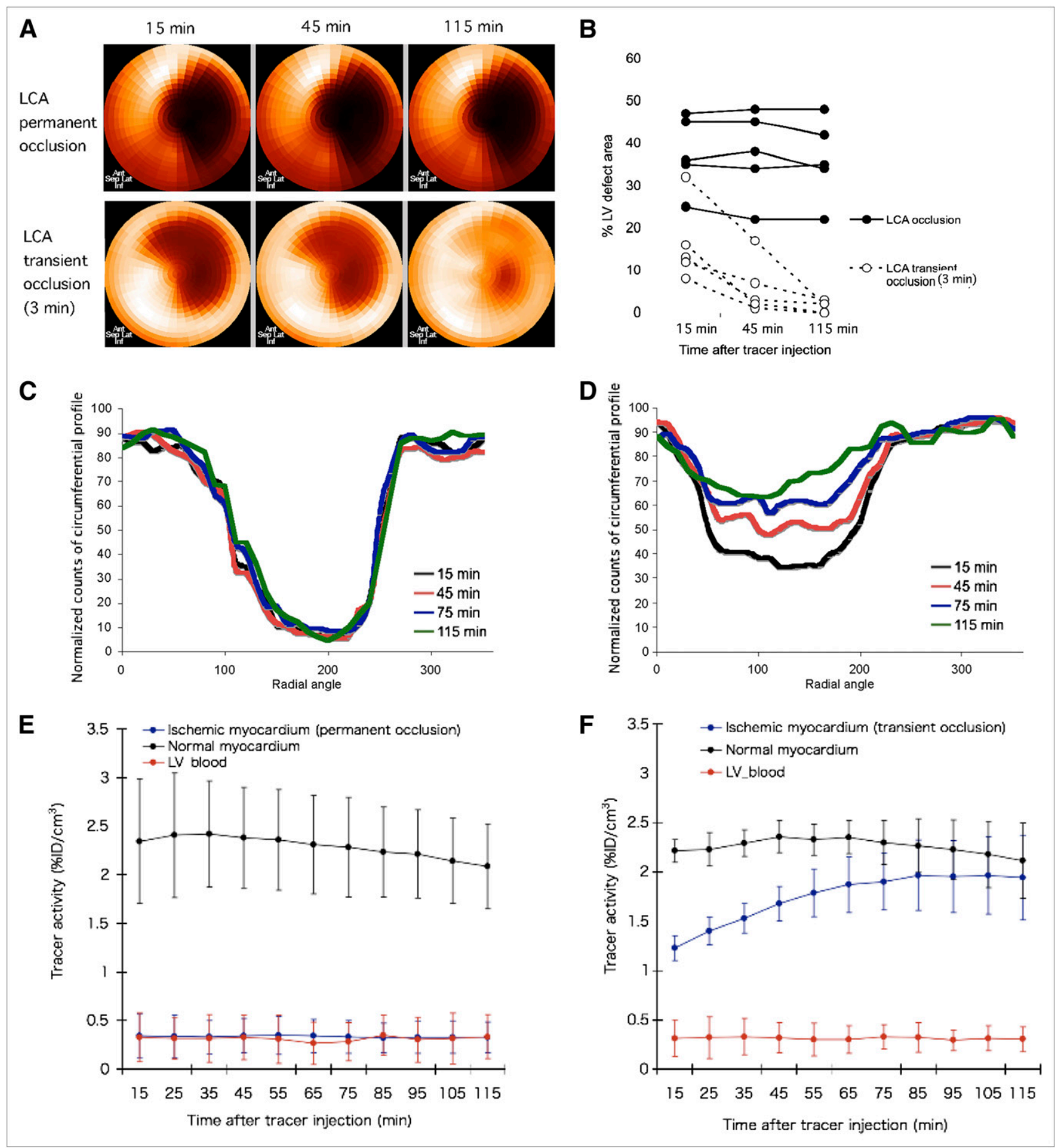

FIGURE 6. Changes in myocardial ${ }^{18} \mathrm{~F}-\mathrm{BMS}-747158-02\left({ }^{18} \mathrm{~F}-\mathrm{BMS}\right)$ distribution after both permanent and transient coronary occlusion are shown in representative polar maps (A) and as defect area expressed as percentage of total left ventricular myocardium (B). Normalized counts of circumferential profiles after permanent occlusion (C) or transient occlusion (D) are also shown. Mean values are shown for tracer activity in induced defect and normally perfused myocardium after permanent (E) and transient occlusion (F). Tracer activity in defect increased over time after reperfusion in transient coronary occlusion.

poxia on tracer retention, which may cause changes in mitochondrial structure and function, have not been addressed. Similar observations have been reported for ${ }^{99 \mathrm{~m}} \mathrm{Tc}-$ sestamibi but have not affected its clinical application $(21,22)$.
Redistribution is an advantage of ${ }^{201} \mathrm{Tl}$ SPECT tracer kinetics that is not shared by ${ }^{99 \mathrm{~m}} \mathrm{Tc}$ perfusion SPECT tracers for the assessment of myocardial viability using stress/rest redistribution protocols. Experimental studies after coronary occlusion and reperfusion in dogs showed 


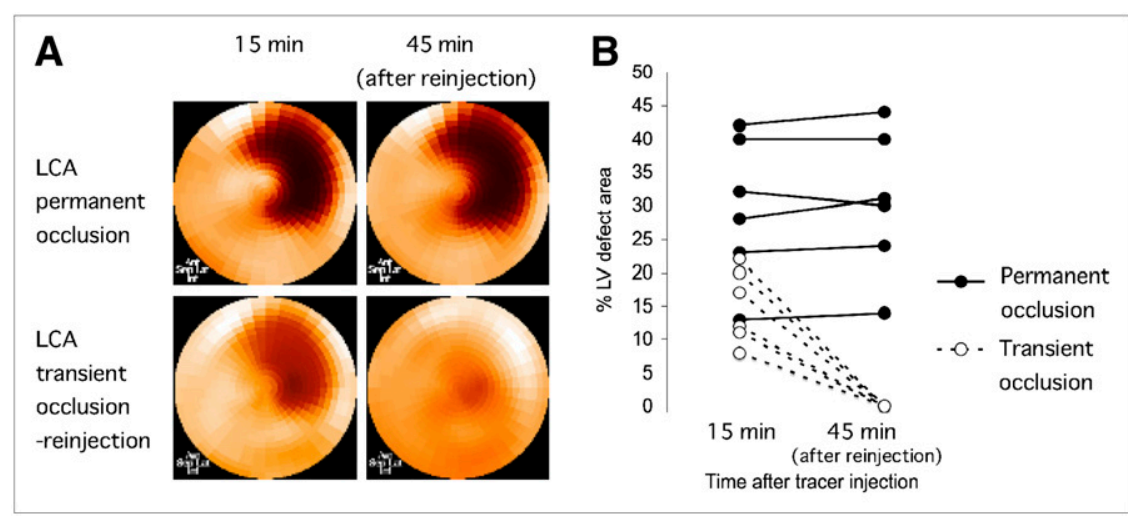

FIGURE 7. Myocardial ${ }^{18} \mathrm{~F}-\mathrm{BMS}-$ 747158-02 (18 $\mathrm{F}-\mathrm{BMS})$ distribution patterns before and after tracer reinjection in rats after permanent and transient coronary occlusion. Representative polar maps $(A)$ and plots of defect area $(B)$ are shown. Tracer uptake defects resolved after tracer reinjection in rats after transient coronary occlusion but not in permanent coronary occlusion. in the induced defect an increase in relative ${ }^{201} \mathrm{Tl}$ activity (23) caused by the differential washout rates in normal and postischemic myocardium. Faster ${ }^{201} \mathrm{Tl}$ washout in normal myocardium results in a decrease in defect size based on the evaluation of relative myocardial tracer distributions.

We observed tracer redistribution with ${ }^{18} \mathrm{~F}-\mathrm{BMS}-$ 747158-02 after reperfusion. In contrast to ${ }^{201} \mathrm{Tl}$ kinetics, we found time-dependent increases in tracer activity in the induced defect whereas the activity remained constant in normally perfused myocardium. High extraction fraction and minimal washout of the tracer from myocardium were demonstrated in isolated perfused rat heart (10). Therefore, after initial tracer uptake, tracer washout from extracardiac organs, as well as some recirculation within the myocardium, may be possible sources of the increased activity. Although the mechanism of redistribution may differ from that of ${ }^{201} \mathrm{Tl}$ redistribution, the observed kinetics may allow for a clinical protocol in the diagnosis of coronary artery disease similar to conventional stress/rest ${ }^{201} \mathrm{Tl}$ perfusion imaging. However, the kinetics observed in rat heart may not directly apply to other species, including humans. Future studies on other species are required to confirm the redistribution and to define the optimal time window between tracer injection and imaging to maintain a high sensitivity for detection of perfusion abnormalities.

\section{CONCLUSION}

We found that cardiac accumulation of ${ }^{18} \mathrm{~F}-\mathrm{BMS}-$ 747158-02 in normal rat hearts was rapid, relatively homogeneous, high, and persisted over $2 \mathrm{~h}$. Additionally, transient coronary occlusion demonstrated well-delineated defects in tracer uptake followed by tracer redistribution after reperfusion. These results indicate that this ${ }^{18} \mathrm{~F}-$ labeled compound may be an excellent candidate in the assessment of myocardial perfusion using PET and conventional physical exercise stress protocols, which are currently used in tandem with SPECT.

\section{ACKNOWLEDGMENTS}

We are grateful to Antti Sarastie and Som Javadi for their careful editorial assistance. This study was supported by a research grant from Bristol-Myers Squibb Medical Imaging. Ming Yu, David S. Casebier, and Simon P. Robinson are employees of Bristol-Myers Squibb Medical Imaging.

\section{REFERENCES}

1. Hachamovitch R, Hayes SW, Friedman JD, Cohen I, Berman DS. A prognostic score for prediction of cardiac mortality risk after adenosine stress myocardial perfusion scintigraphy. J Am Coll Cardiol. 2005;45:722-729.

2. Lima RS, Watson DD, Goode AR, et al. Incremental value of combined perfusion and function over perfusion alone by gated SPECT myocardial perfusion imaging for detection of severe three-vessel coronary artery disease. J Am Coll Cardiol. 2003;42:64-70.

3. Simons M, Bonow RO, Chronos NA, et al. Clinical trials in coronary angiogenesis: issues, problems, consensus: an expert panel summary. Circulation. 2000;102:E73-E86.

4. Di Carli MF, Hachamovitch R. Should PET replace SPECT for evaluating CAD? The end of the beginning. J Nucl Cardiol. 2006;13:2-7.

5. Bateman TM, Heller GV, McGhie AI, et al. Diagnostic accuracy of rest/stress ECG-gated Rb-82 myocardial perfusion PET: comparison with ECG-gated Tc99m sestamibi SPECT. J Nucl Cardiol. 2006;13:24-33.

6. Madar I, Ravert HT, Du Y, et al. Characterization of uptake of the new PET imaging compound ${ }^{18} \mathrm{~F}$-fluorobenzyl triphenyl phosphonium in dog myocardium. J Nucl Med. 2006;47:1359-1366.

7. Marshall RC, Powers-Risius P, Reutter BW, et al. Kinetic analysis of ${ }^{18} \mathrm{~F}-$ fluorodihydrorotenone as a deposited myocardial flow tracer: comparison to ${ }^{201}$ Tl. J Nucl Med. 2004;45:1950-1959.

8. Yu M, Guaraldi MT, Mistry M, et al. BMS-747158-02: a novel PET myocardial perfusion imaging agent. J Nucl Cardiol. 2007;14:789-798.

9. Yalamanchili P, Wexler E, Hayes M, et al. Mechanism of uptake and retention of F-18 BMS-747158-02 in cardiomyocytes: a novel PET myocardial imaging agent. J Nucl Cardiol. 2007;14:782-788.

10. Huisman MC, Higuchi T, Reder S, et al. Initial characterization of an ${ }^{18} \mathrm{~F}$-labeled myocardial perfusion tracer. J Nucl Med. 2008;49:630-636.

11. Levin CS, Hoffman EJ. Calculation of positron range and its effect on the fundamental limit of positron emission tomography system spatial resolution. Phys Med Biol. 1999;44:781-799.

12. Sanchez-Crespo A, Andreo P, Larsson SA. Positron flight in human tissues and its influence on PET image spatial resolution. Eur J Nucl Med Mol Imaging. 2004;31:44-51.

13. Guide for the Care and Use of Laboratory Animals. Washington, DC: National Academy Press; 1996.

14. Higuchi T, Nekolla SG, Jankaukas A, et al. Characterization of normal and infarcted rat myocardium using a combination of small-animal PET and clinical MRI. J Nucl Med. 2007;48:288-294.

15. Higuchi T, Taki J, Nakajima K, Kinuya S, Namura M, Tonami N. Time course of discordant BMIPP and thallium uptake after ischemia and reperfusion in a rat model. J Nucl Med. 2005;46:172-175.

16. Nekolla SG, Miethaner C, Nguyen N, Ziegler SI, Schwaiger M. Reproducibility of polar map generation and assessment of defect severity and extent assessment in myocardial perfusion imaging using positron emission tomography. Eur $J$ Nucl Med. 1998;25:1313-1321.

17. Marwick TH, Go RT, MacIntyre WJ, Saha GB, Underwood DA. Myocardial perfusion imaging with positron emission tomography and single photon 
emission computed tomography: frequency and causes of disparate results. Eur Heart J. 1991;12:1064-1069.

18. Beanlands RS, Chow BJ, Dick A, et al. CCS/CAR/CANM/CNCS/CanSCMR joint position statement on advanced noninvasive cardiac imaging using positron emission tomography, magnetic resonance imaging and multidetector computed tomographic angiography in the diagnosis and evaluation of ischemic heart disease: executive summary. Can J Cardiol. 2007;23:107-119.

19. Chow BJ, Beanlands RS, Lee A, et al. Treadmill exercise produces larger perfusion defects than dipyridamole stress N-13 ammonia positron emission tomography. J Am Coll Cardiol. 2006;47:411-416.

20. Schuler F, Casida JE. The insecticide target in the PSST subunit of complex I. Pest Manag Sci. 2001;57:932-940.
21. Weinstein H, Reinhardt CP, Wironen JF, Leppo JA. Myocardial uptake of thallium 201 and technetium 99m-labeled sestamibi after ischemia and reperfusion: comparison by quantitative dual-tracer autoradiography in rabbits. J Nucl Cardiol. 1994;1:351-364.

22. Meerdink DJ, Leppo JA. Comparison of hypoxia and ouabain effects on the myocardial uptake kinetics of technetium-99m hexakis 2methoxyisobutyl isonitrile and thallium-201. J Nucl Med. 1989;30:15001506.

23. Pohost GM, Zir LM, Moore RH, McKusick KA, Guiney TE, Beller GA. Differentiation of transiently ischemic from infarcted myocardium by serial imaging after a single dose of thallium-201. Circulation. 1977;55: 294-302.

\section{Errata}

In the article "Direct Imaging of Radionuclide-Produced Electrons and Positrons with an Ultrathin Phosphor," by Chen et al. (J Nucl Med. 2008;49:1141-1145), reference 11 was cited incorrectly. The correct citation is as follows: Cho JS, Vu NT, Chung YH, et al. Detection of beta particles in a microfluidic chip using a scintillator and CCD. In: 2006 IEEE Nuclear Science Symposium Conference Record. Piscataway, NJ: IEEE; 2006:1977-1981. The authors regret the error.

The authors of "Combination Therapy and Noninvasive Imaging with a Dual Therapeutic Vector Expressing MDR1 Short Hairpin RNA and a Sodium Iodide Symporter," by Park et al. (J Nucl Med. 2008;49:1480-1488), would like to rectify a mistake in the surname of the third author of the article. The correct surname is Thapa. The authors regret the error. 